\title{
Losing weight with SODA: the impact of environment on disk properties in Orion A
}

Sierk van Terwisga ${ }^{1}$, Alvaro Hacar ${ }^{2}$, and Ewine van Dishoeck ${ }^{2,3}$

${ }^{1}$ Max Planck Institute for Astronomy, Germany (terwisga@mpia.de)

${ }^{2}$ Leiden Observatory, The Netherlands

${ }^{3}$ Max-Planck-Institut für Extraterrestrische Physik, Germany

\begin{abstract}
The evolution of millimeter-sized dust in a protoplanetary disk is one of the key observable constrains on the formation of planets. In recent years, ALMA surveys of disks in nearby starforming regions have shown a dependence of the disk mass distribution on cloud properties and environmental factors. Due to the relatively small number of regions for which these statistics are available, several key questions have so far been out of reach for observers: do disk masses vary across clouds, or are they constant? How far does the influence of massive young stars reach in different environments? How do the masses of Class II-disks compare to those of embedded objects?
\end{abstract}

We present new results from the Survey of Orion Disks with ALMA (SODA), based on observations of 882 disks in the southern part of the Orion A molecular cloud. This flux-limited survey is the largest of its kind so far, and spans a large area on the sky with multiple young groups and clusters, as shown in Figure 1. We find that disk properties across the cloud are remarkably uniform, between clusters and between regions of different stellar density. However, the presence of B-stars is significant.

\section{Introduction}

Different ALMA surveys in individual star forming regions have significantly improved our understanding of the evolution of protoplanetary disks in recent times. In particular, most of the nearby low-mass star-forming regions (SFRs) have now been observed at mm-wavelengths, as well as several SFRs hosting massive stars (e.g., Ansdell et al. 2016, Pascucci et al. 2016, Eisner et al. 2018, van Terwisga et al. 2020). These studies have provided key insight in how circumstellar matter evolves as planetary systems take shape.

Observations so far have shown that disks of all masses evolve rapidy within the first few Myr of their existence (Tychoniec et al. 2020). In addition to these age effects, the disk mass distribution of populations of disks in strongly irradiated environments has also been shown to be subject to external photoevaporation. Disks decrease in mass in stronger radiation fields in regions like $\sigma$ Ori and the Trapezium (Ansdell et al. 2017, Mann et al. 2014, Eisner et al. 2018), demonstrating the direct impact of extreme environments on disk evolution. Model predictions (from e.g. the FRIED grid, by Haworth et al. 2018) and observations of individual disks in low-mass SFRs have shown that even in an environment like Taurus, where the UV radiation is a factor 1000 
weaker than in the Trapezium, half of the disks undergo significant mass loss.

A key problem is that even with the current state-of-the-art data, the number of different starforming regions sampled is quite small. Additionally, even in regions where complete surveys of the Class II population exist, like Lupus, stars exist in a range of environmental conditions. Our survey of Orion A, the largest of its kind so far, covers large numbers of disks in a wide variety of environments, allowing us to link disk properties to their enviroment more firmly than before.

Figure 1: The 882 Class II YSOs targeted in this survey (green plus signs), and groups and clusters identified by Megeath et al. 2016 (pink crosses). The background shows the total gas and dust column as derived from Herschel continuum maps (Lombardi et al. 2014).

\section{Data reduction}

Our observations cover a large number of sources at low (1.4") resolution in ALMA Band 6. This resolution is low compared to the expected disk structure. This meant we could use a highperformance computing cluster to reduce and image the data in a fraction of the time it would otherwise take, simulateously guaranteeing consistent and reproducible results.

\section{Results}

Figure 2: Disk mass distribution in the SODA sample, compared to several other well-studied nearby star-forming regions.

By converting disk fluxes to masses we can compare the properties of the Orion A disks sample, both within different regions of Orion $A$, and between Orion $A$ and other clouds, like Lupus and Taurus, which have been surveyed previously. As Figure 2 shows, the large number of disks in our sample allows us to determine the disk mass distribution much more accurately than previously possible. However, the large sample size also enables us to compare average disk masses between regions in Orion $A$, as shown in Figure 3, which shows that there are only small differences in average disk masses between areas of vastly different surface densities, even across the length of the cloud, and emphasizes the remarkable similarity between disks in Orion, Taurus, and Lupus. However, the presence of nearby B-stars is relevant for disk masses, out to scales of several parsecs, a parameter range that it was not previously possible to probe.

Figure 3: Disk masses as a function of density of YSOs, across Orion A, and in Lupus, show a remarkable similarity across many orders of magnitude. 